\section{Examen spécial en vue de l'obtention du titre de spécialiste FMH en médecine physique et réadaptation pour les chefs de cliniques de réhabilitation reconnues, conformément aux dispositions transitoires du RFP PMR}

Le programme de formation postgraduée entré en vigueur le $1^{\mathrm{er}}$ janvier 2000 et la nouvelle version des dispositions transitoires approuvées le 23 avril 2001 par le comité central de la FMH prévoient les conditions suivantes à l'obtention du titre de spécialiste en "médecine physique et réadaptation». Peuvent obtenir cette reconnaissance les personnes possédant le titre de spécialiste FMH, ayant dirigé avant le $1^{\text {er }}$ janvier 2000 pendant au moins 5 ans une clinique de réadaptation reconnue dans les domaines ci-dessous et qui dirigent encore une telle clinique au moment du dépôt de la demande:

- réadaptation pour les maladies neurologiques,

- réadaptation pour les maladies pneumologiques,

- réadaptation pour les maladies cardiologiques,

- réadaptation pour les maladies pédiatriques,

- réadaptation pour les maladies musculosquelettales (réadaptation rhumatologique, orthopédique et post-traumatique).

La durée de 5 ans peut être réduite en fonction des années de formation postgraduée spécifique reconnue que le candidat peut faire valoir.

La clinique/division doit en outre remplir les critères suivants:

- elle doit être dirigée par un médecin-chef ou un médecin dirigeant à plein temps;

- elle doit posséder une division séparée du point de vue organisationnel, personnel et spatial, contenant au moins 25 lits réservés pour la réadaptation;
- elle doit avoir un personnel soignant à temps complet dont la formation correspond au moins au diplôme de niveau D1;

- elle doit avoir des physiothérapeutes diplômés avec un statut d'employés;

- au moment du dépôt de la demande, la clinique doit figurer sur la liste des hôpitaux du canton d'accueil ou du canton subventionneur avec un mandat spécifique pour la réadaptation;

- la clinique/division doit être reconnue par la FMH comme un centre de formation postgraduée au moment du dépôt de la demande.

La SSMPR peut ordonner une visite de l'établissement pour contrôler le respect de ces critères. Le cas échéant, les frais sont à la charge du demandeur.

\section{Premier examen oral}

Lieu: Clinique St. Anna, Physikalische Therapie, Lucerne

Date: mercredi 12 septembre 2001

Taxe d'examen: La Société Suisse de Médecine Physique et Réadaptation demande une participation aux frais de Fr. 500.- pour l'examen oral.

Inscriptions: Secrétariat SSMPR, c/o Ligue suisse contre le rhumatisme, Renggerstrasse 71, $8038 \mathrm{Zu}$ rich, tél. 0148740 00/07, fax 0148740 19, e-mail: info@rheuma-net.ch

Titre

Le titre de spécialiste FMH en médecine physique et réadaptation est octroyé dès que le candidat peut faire valoir que l'examen oral spécial a été passé avec succès.

Délai d'inscription: 31 juillet 2001

Conformément à ces dispositions transitoires, la demande d'obtention du titre de spécialiste FMH en médecine physique et réadaptation doit être remise avant le 31 décembre 2001. 